\title{
High permeability flexible bulk material for magnetic micro head applications
}

\author{
A. Belski, P. Taptimthong, M. C. Wurz, and L. Rissing \\ Institute for Micro Production Technology, Leibniz Universität Hannover, 30823 Garbsen, Germany
}

\begin{abstract}
A magnetic field is generated by feeding electric current to micro coils. The higher the current, the higher the magnetic field is. A main disadvantage of coils in micro systems is the limited thermal resistance and as a result of a restriction concerning the maximum current densities. This work shows the modelling, design, numerical calculation and the initial test of a flexible write head, which not only allows the data storage on the surface but also inside of the components. Furthermore, the write head has to be flexible and able to adapt itself to the roughness of the component surface. The main goal of this paper is to increase the magnetic flux in the micro head core by partly using high permeability flexible bulk material instead of increasing the coil current or the number of the coil turns. For the approval of the data storage process a GMR sensor is used for the readout of the stored magnetization track.
\end{abstract}

\section{Introduction}

The term "gentelligent" describes a new feature of the inherent combination of a component and its corresponding information, which includes the geometric values or information that is generated during manufacturing or usage [1]. This information can be used for further development, maintenance or for components' identification. The storage material of the components is "magnetic magnesium", which is $\mathrm{Mg}$ with embedded hard magnetic $\gamma-\mathrm{Fe}_{2} \mathrm{O}_{3}$ particles. For an application of data storage directly on the components an adapted magnetic recording head is developed and fabricated. The storage surface of the gentelligent components varies depending on different geometries. Therefore a flexible writing head is required which adapts its own geometry to the surface topography during data storage process. This recording head uses a special design which allows the application of flexible $\mathrm{NiFe}, \mathrm{Cu}$ and polymer foils in the micro head structure. The chosen highly magnetic permeability bulk material is a $\mathrm{NiFe}$ foil with $70-80 \% \mathrm{Ni}$, which serves as one part of the flexible flux guide.

\section{Concept}

The flexible magnetic micro head consists of a magnetic core, coils and an air gap. Two micro coils induce a magnetic flux into the magnetic core material. The soft magnetic core guides the magnetic flux to the air gap, where the spreading magnetic field magnetizes a gentelligent material surface. Figure 1 shows a schematic view of the flexible magnetic head.

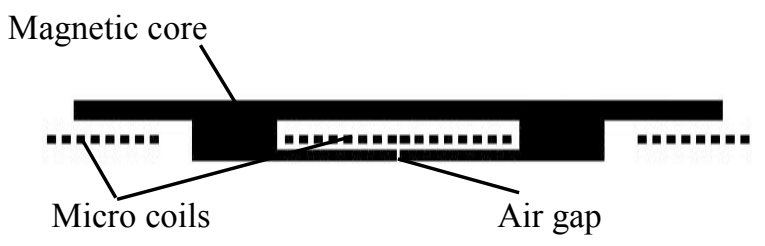

Fig. 1. Flexible magnetic write head

The high magnetic field intensity in the air gap requires high current in the micro coils. The main disadvantage of this approach is the limitation of the maximum current value that generates excessive heat damaging the micro device. The magnetic reluctance of the core $R_{m}$ can be expressed as

$$
R_{m}=\frac{l}{\mu \cdot A}
$$

where $l$ is the length, $\mu$ is the permeability, and $A$ is the cross section of the magnetic core. The increasing relative permeability of the core material decreases the magnetic reluctance. The magnetic flux $\Phi$ is also affected by magnetic reluctance which can be defined as:

$$
\Phi=\frac{F}{R_{m}}
$$


where $F$ is the magnetomotive force. The increasing relative permeability of the core material increases the magnetic flux. The magnetic flux density $B_{g}$ in the air gap is:

$$
B_{g}=\frac{\Phi}{A}
$$

where $A$ is the cross section of the gap. The required high flux density in the air gap for the magnetization of the gentelligent components can be achieved by increasing the magnetic flux and reduction of the cross section area of the gap, which leads to the reduction of the bit size in the track and to an increase of storage density. The equations 1-3 show that the spreading field in the air gap can be also maximized by increasing the relative permeability of the magnetic core material. For simplification of the thin film fabrication, the high permeability bulk material in the magnetic head structure is only partly integrated. In this case the commercial NiFe foil with the relative permeability of 50,000 and saturation flux density $B_{s}$ of $0.8 \mathrm{~T}$ is used. The NiFe foil is integrated by the adhesion process as a top flux guide of the magnetic core as shown in figure 2 .

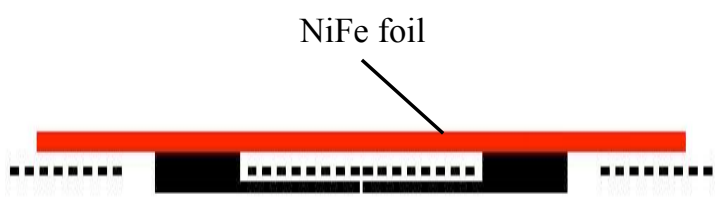

Fig. 2. Write head with integrated high permeability NiFe foil

\section{Head Geometry}

The first prototype of the magnetic head is fabricated in an ultra-precision dicing process and creates bits with a width of $700 \mu \mathrm{m}$ and a length of a $100 \mu \mathrm{m}$ [2]. The flexible magnetic write head is fabricated by thin film technology and allows creating magnetic tracks with a width of $200 \mu \mathrm{m}$ and a length of $10 \mu \mathrm{m}$. Figure 3 contains the schematic representation of the flexible magnetic write head.

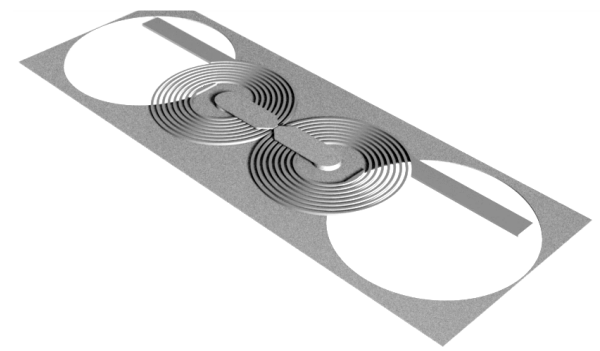

Fig. 3. Flexible magnetic write head

The thin film fabrication processes require special boundary conditions for the head geometry. In the following the head geometry is applied with respect to the process conditions. The cross section of the head gap in the bottom flux guide is $20 \mu \mathrm{m} \times 200 \mu \mathrm{m}$. The NiFe foil which is used as the top flux guide has a thickness of $50 \mu \mathrm{m}$. The poles have a cylinder form with the diameter of $1 \mathrm{~mm}$. The bottom flux guide of the core has a thickness of $20 \mu \mathrm{m}$. The poles and the bottom flux guide are fabricated by electroplating of $\mathrm{NiFe} 81 / 19$, which has the saturation flux density $B_{s}$ of $1.0 \mathrm{~T}$. The cross section of the copper coil winding is $100 \mu \mathrm{m} \times 17.5 \mu \mathrm{m}$. The whole head structure has a footprint of $25 \mathrm{~mm} \times 10 \mathrm{~mm}$. Due to the head design the required number of the coil turns is eight.

\section{Numerical Calculations}

An evaluation of the recording head by a numerical Finite Element Method (FEM) is carried out with the simulation software ANSYS ${ }^{\mathrm{TM}}$. As the data is recorded horizontally, the horizontal component of the writing field $H_{x}(x, y)$ shall be analyzed according to Karlqvist [3-4], with the boundary condition:

$$
H_{x}(x, y)>H_{c, \text { material }}
$$

where $H_{c, \text { material }}$ represents the coercivity of the storage medium. The coercivity $H_{c}$ of the hard magnetic $\gamma-\mathrm{Fe}_{2} \mathrm{O}_{3}$ particles is $23 \mathrm{kA} / \mathrm{m}$. For the construction of the magnetic head, the Karlqvist condition is met. To avoid the saturation effect in the magnetic core, a simulation of the magnetic flux density with eight coil turns is carried out. The maximal values of the flux density in the $\mathrm{NiFe}$ foil and NiFe81/19 are shown depending on the coil current are shown in figure 4 . The coil current of $800 \mathrm{~mA}$ is sufficient for both parts of the magnetic core.

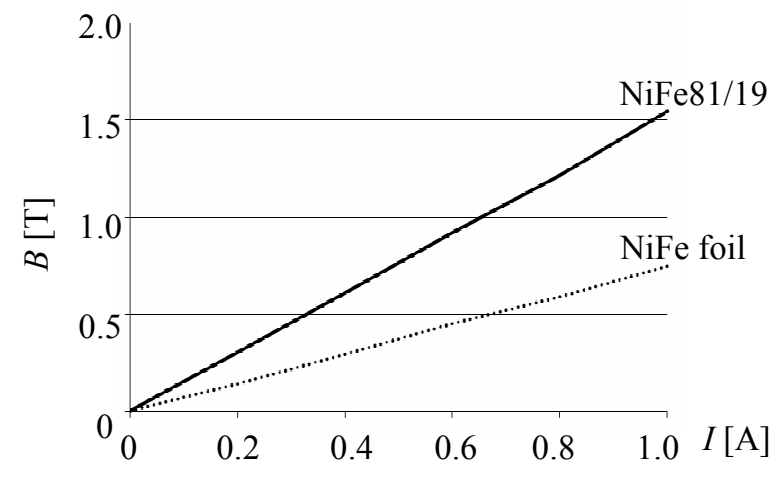

Fig. 4. FEM simulation for the magnetic flux density in the magnetic core

The initial test for the maximum current in the micro coils is done. Due to the thermal load the coil current has to be decreased and set to $600 \mathrm{~mA}$. This value is used in all numerical simulations. The numerical calculations with ANSYS are carried out in two steps. In the first step the FEM simulation is executed for the micro structure with the magnetic core material from NiFe81/19. The polymer foil can be coated with the NiFe81/19 by electroplating process and has the relative permeability of 3,000 [5]. In figure 5, the distribution of the magnetic field intensity $H_{x}$ is represented. It shows the Karlqvist field, which is simulated by using only NiFe81/19 as the magnetic core material in the whole microstructure. Due 
to the Karlqvist boundary condition, the number of the coils has to be set to ten in this simulation. The generated magnetic field strength $H_{x}$ in the middle of the air gap, where the magnetic field spreads out to the component's surface area, is $140 \mathrm{kA} / \mathrm{m}$. The gentelligent components require a depth magnetization minimum of $10 \mu \mathrm{m}$. With this writing head, it is possible to magnetize storage material, up to a depth of $10 \mu \mathrm{m}$.

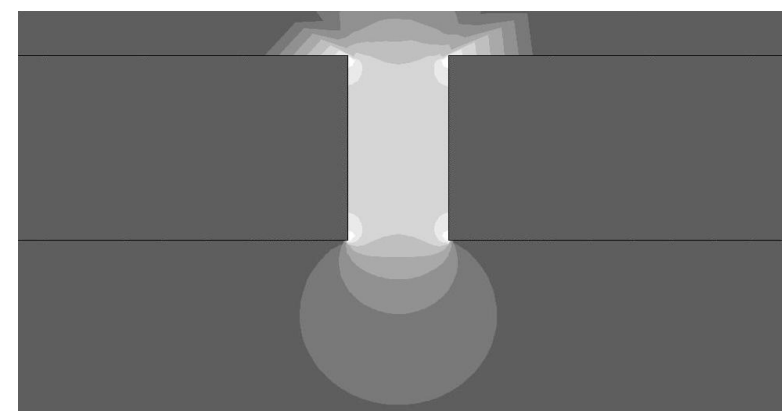

$$
\begin{array}{cccccccc}
30 & 60 & 90 & 120 & 150 & 180 & 210 & 240 \\
& & H x & {[\mathrm{kA} / \mathrm{m}]}
\end{array}
$$

Fig. 5. FEM simulation for the air gap with the magnetic core relative permeability of 3,000 and 10 coil turns

In the second step the simulation is carried out with $\mathrm{NiFe} 81 / 19$ as a lower core with a relative permeability of 3,000 and $\mathrm{NiFe} 80 / 20$ as the upper core with a relative permeability of 50,000 . The number of the coil turns is decreased to eight. The distribution of the magnetic field intensity $H_{x}$ in the air gap of the write head is presented in figure 6 . In this case, where the magnetic core part uses the high permeability material with eight coil turns, the Karlqvist boundary condition is still satisfied.

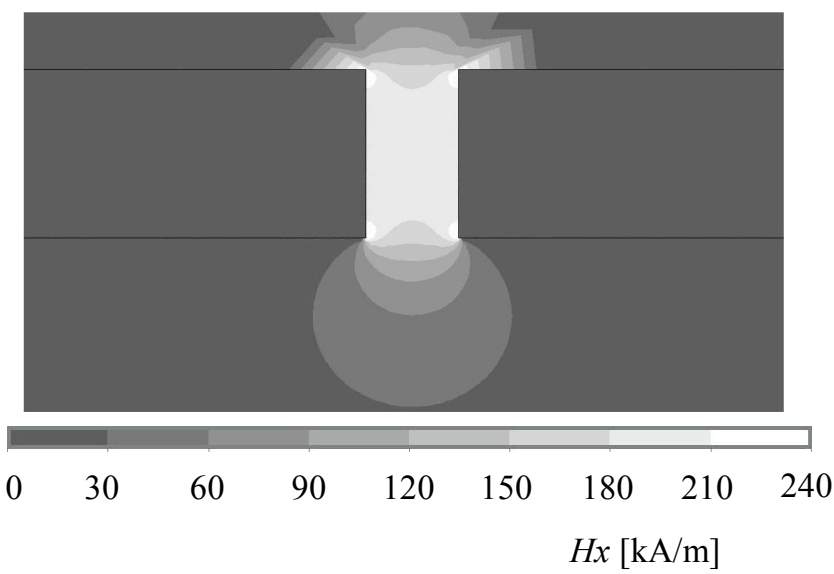

Fig. 6. FEM simulation for the air gap with the magnetic core relative permeability of 3,000 and 50,000 and 8 coil turns

The spreading field on the contact point with the surface increases to the $160 \mathrm{kA} / \mathrm{m}$. Furthermore, this micro head allows magnetizing of the storage material, up to a depth of $12 \mu \mathrm{m}$. The magnetization into the depth of the material is increased by use of high permeability bulk material. In the case of the relative permeability of 50,000 by using only flexible NiFe bulk material as the magnetic core material in the whole microstructure and the coil numbers is eight, the spreading field increases to the $220 \mathrm{kA} / \mathrm{m}$. The magnetization depth increases up to the $15 \mu \mathrm{m}$.

\section{Experimental}

Magnetic $\mathrm{Mg}$ is a sintered material. A planarization process of its surface requires a polishing process which is only feasible to a limited extent due to the brittleness of the sintered material so far. Therefore, for the first experimental results an alternative material to magnetic $\mathrm{Mg}$ is used. The data storage process is carried out on the magnetic tape from a compact cassette, which is coated with $\gamma-\mathrm{Fe}_{2} \mathrm{O}_{3}$ layer and has the same magnetic properties as the magnetic $\mathrm{Mg}$ samples. For the experimental results the magnetic write head is fixed with the air gap upwards (figure 7).

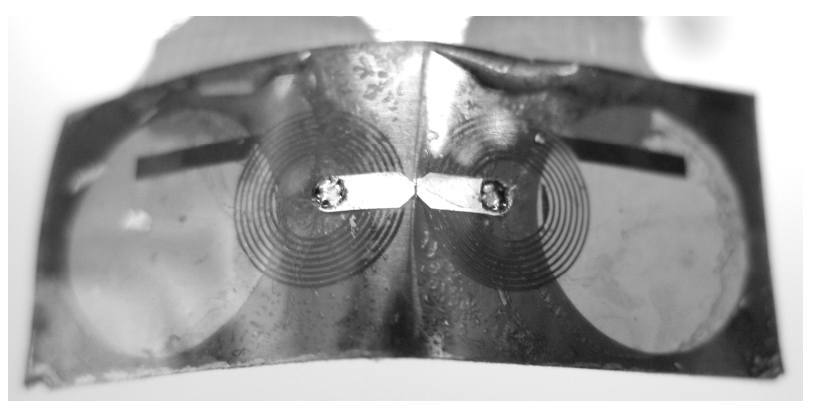

Fig. 7. Magnetic write head

To prove that only the magnetic tracks from the write head are detected after the storage process, all previous magnetization states have to be deleted. For this aim to be achieved, the magnetic tape is demagnetized. Afterwards it is fixed between two carriers. The $\gamma-\mathrm{Fe}_{2} \mathrm{O}_{3}$ layer is positioned downwards to the air gap of the head (figure 8). The head is guided over the $\gamma-\mathrm{Fe}_{2} \mathrm{O}_{3}$ surface by the electrical drive. Both coils of the head are fed with a current of $600 \mathrm{~mA}$. The alternating sequence of " 0 " and " 1 " is created on the tape surface.

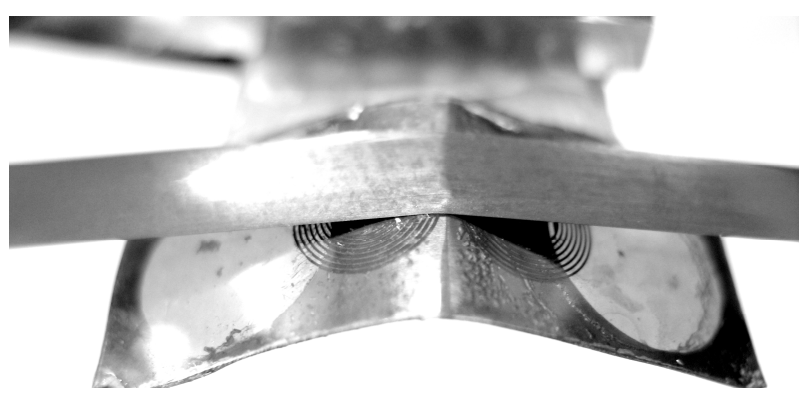

Fig. 8. Magnetic tape over the write head.

For the evaluation of the magnetic tracks, the magnetic field sensor based on the multilayer Giant Magneto Resistive (GMR) effect is used. The commercial GMR sensor consists of a Wheatstone bridge with on-chip flux concentrators for enhancing of sensitivity. The output 
signal of the device is the DC voltage which depends on the applied magnetic field. After the storage process the magnetic tape is mounted by double sided adhesive tape on a glass plate. The GMR sensor is fixed on the carrier and guided over the surface of the magnetic tape surface controlled by the same electrical drive unit as the write head (figure 9).

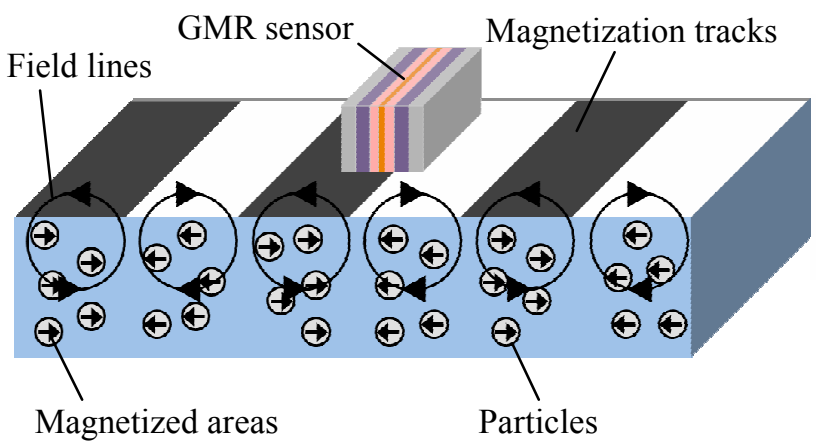

Fig. 9. GMR sensor over the magnetic tape

The output signal of the readout process is presented in figure 10 . For analyzing the output signal, special signal analyzing techniques as determination of the gradient, determination of extremum, curve analysis and state machine are used [6].

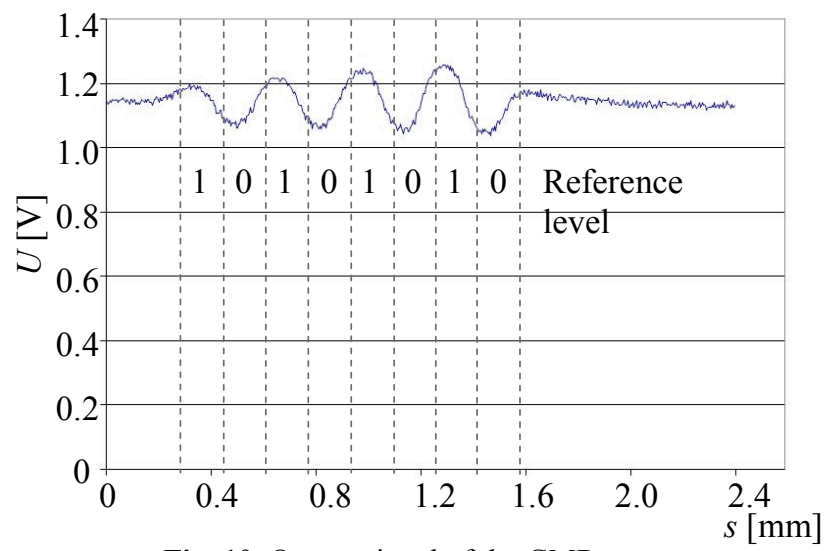

Fig. 10. Output signal of the GMR sensor

For the investigation of the head behaviour during the bending a magnetometer is used. Due to the construction of the magnetometer, the magnetic field could be measured $400 \mu \mathrm{m}$ under the air gap. This explains the small values for the magnetic field intensity (table 1). The measurements are carried out for the followed bending angle of the head: $0^{\circ}, 50^{\circ}$, and $90^{\circ}$. Due to the decreasing of the magnetic core permeability regarding to the mechanical stress, the magnetic field intensity under the air gap decreases by increasing of the bending angle.

Tab. 1. Dependence of the field intensity on bending angle

\begin{tabular}{|c|c|c|c|}
\hline \multirow{2}{*}{$\begin{array}{c}\text { Current } I \\
{[\mathrm{~A}]}\end{array}$} & \multicolumn{3}{|c|}{ Magnetic field intensity $H[\mathrm{kA} / \mathrm{m}]$} \\
\cline { 2 - 4 } & $0^{\circ}$ & $50^{\circ}$ & $90^{\circ}$ \\
\hline 0.40 & 0.27 & 0.20 & 0.14 \\
\hline 0.45 & 0.32 & 0.24 & 0.16 \\
\hline 0.50 & 0.37 & 0.27 & 0.18 \\
\hline 0.60 & 0.46 & 0.35 & 0.24 \\
\hline
\end{tabular}

\section{Conclusions}

The stored alternating sequence of "10101010" is successfully detected. A proof for the functionality of the flexible write head is achieved. The head is able to magnetize the $\gamma-\mathrm{Fe}_{2} \mathrm{O}_{3}$ hard magnetic particles which proves the accuracy of the head design. The application of the high permeability flexible bulk material shows good results. Due to an enhancement effect of the high permeability materials it is possible to simplify the micro systems and to avoid multilayer constructions. The eight coil turns are enough to satisfy the Karlqvist boundary condition for data storage on the particle based medium. Furthermore, the thermal load of the micro coil can be reduced. The future goals are to decrease the size of the write head and to investigate the flexible properties of write head on the magnetic Mg surface.

\section{Acknowledgements}

This research is sponsored in part by the DFG (German Research Foundation) within the SFB (Collaborative Research Center) 653 "Gentelligent Components in their Lifecycle".

\section{References}

1. B. Denkena, H. Henning, L.E. Lorenzen, J. Prod. Eng., Genetics and intelligence: new approaches in production engineering, 4, 65-73 (2010)

2. A. Belski, M.C. Wurz, L. Rissing, EUSPEN 11 Conf. Proc., Redesign and fabrication of a magnetic head for gentelligent products, 2, 168-171 (2011)

3. K.G. Ashar, Magnetic disk drive technology, (IEEE Press, 1997)

4. O. Karlqvist, Trans. R. Inst. Technol., Calculation of the magnetic field in the ferromagnetic layer of $a$ magnetic drum, 86, 3-27 (1954)

5. J. Chen, M. C. Wurz, A. Belski, L. Rissing, IEEE Trans. on Magn., Designs and characterizations of soft magnetic flux guides in a 3-D magnetic field sensor, 48, 1481-1484 (2012)

6. A. Belski, P. Taptimthong, M.C. Wurz, L. Rissing, SysInt, Integration of the read-after-write method for gentelligent applications, 1, 49-51 (2012) 